\title{
Assessment of psychological barriers to cervical cancer screening among women in Kumasi, Ghana using a mixed methods approach
}

\author{
*Williams $\mathrm{M}^{1}$, Kuffour $\mathrm{G}^{2}$, Ekuadzi $\mathrm{E}^{3}$, Yeboah $\mathrm{M}^{4}$, ElDuah $\mathrm{M}^{1}$, Tuffour $\mathrm{P}^{2}$
}

1. Department of Health Behavior, University of Alabama at Birmingham, USA

2. Department of Pharmaceutics, Faculty of Pharmacy and Pharmaceutical Sciences Kwame Nkrumah University of Science and Technology, Ghana

3. Department of Pharmacognosy, Faculty of Pharmacy and Pharmaceutical Sciences Kwame Nkrumah University of Science and Technology, Ghana

4. Department of Pharmaceutical Sciences, Mercer University, USA

\begin{abstract}
Background: Cervical cancer is the leading cause of cancer death among women in Ghana, West Africa. The cervical cancer mortality rate in Ghana is more than three times the global cervical cancer mortality rate. Pap tests and visual inspection with acetic acid wash are widely available throughout Ghana, yet less that $3 \%$ of Ghanaian women get a cervical cancer screening at regular intervals.

Objective: This exploratory study was to identify psychological barriers to cervical cancer screening among Ghanaian women with and without cancer using a mixed methods approach.

Methods: Semi-structured interviews were conducted with 49 Ghanaian women with cancer and 171 Ghanaian women who did not have cancer.

Results: The results of the quantitative analysis indicated that cancer patients where not more likely to have greater knowledge of cancer signs and symptoms than women without cancer. Analysis of the qualitative data revealed several psychological barriers to cervical cancer screening including, common myths about cervical cancer, misconceptions about cervical cancer screening, the lack of spousal support for screening, cultural taboos regarding the gender of healthcare providers, and the stigmatization of women with cervical cancer.

Conclusion: The results of this study can be used to inform the development of culturally relevant cervical cancer education interventions aimed at addressing the psychological barriers to cervical cancer screening perceived by Ghanaian women.

Keywords: Cancer, developing countries, disease prevention, knowledge, qualitative methods

African Health Sciences 2013; 13(4): 1054 - 1061 http://dx.doi.org/10.4314/ahs.v13i4.28
\end{abstract}

\section{Introduction}

Cervical cancer is the second most common cancer that affects women worldwide ${ }^{1}$. In Ghana, West Africa, cervical cancer is the leading cause of cancer death among women ${ }^{1-3}$. It is estimated that every year, more than 3,000 Ghanaian women develop cervical cancer and more than 2,000 Ghanaian women die because of the disease ${ }^{1}$. The cumulative risk of Ghanaian women dying from cervical cancer is nearly three times the global cumulative risk (3.3\% vs. $0.9 \%$, respectively) ${ }^{1}$. Researchers at the World Health Organization (WHO) ${ }^{1}$ have predicted that by

* Corresponding author:
Michelle S. Williams
University of Alabama at Birmingham
School of Public Health, Ryals Public Health
Building
Room 227, 1665
University Boulevard, Birmingham, AL 35294-00
Telephone: (205) 515-9156
Email: msw117@uab.edu

2025, there will be approximately 5,007 new cases of cervical cancer and 3,361 deaths due to cervical cancer annually in Ghana.

Experts in the field of oncology have suggested that cervical cancer can be eradicated through preventive screening and the early detection of precancerous and cancerous lesions ${ }^{4-8}$. In addition to the Human Papillomavirus (HPV) vaccines and HPV DNA testing, there are screening tools that can be used help reduce the cervical cancer burden ${ }^{6,9}$. The cytology-based Pap test is the most commonly used cervical cancer screening method in Ghana. Visual inspection with acetic acid wash (VIA) is an efficient cervical cancer screening tool that has been used in urban and rural areas in Ghana ${ }^{10,11}$. The Pap test and VIA are available at a healthcare facilities throughout the country. Despite the availability of these cervical cancer-screening tools, only $2.7 \%$ of Ghanaian women between the ages of $15-64$ obtain cervical cancer screenings every 3 years ${ }^{1}$. 
Ghanaian healthcare providers have indicated that approximately $70 \%$ of the patients who are diagnosed with cancer are diagnosed when their tumors have progressed to late stages at which point they are very difficult to treat ${ }^{12-14}$. For example, case studies of Ghanaian cervical cancer patients indicate that some women with cervical cancer delayed seeking treatment until their cervical cancer tumors metastasized and developed on their abdomens ${ }^{15}$. Psychological barriers may be significant impediments to preventive healthcare behaviors and treatmentseeking behaviors ${ }^{15}$. These psychological barriers include perceived invulnerability, lack of knowledge, and lack of social support ${ }^{15}$.

The Ghana Health Ministry has called for a reduction in cervical cancer mortality ${ }^{16}$. There is no evidence that significant actions have been taken to increase the Ghanaian women's awareness of the risk factors for cervical cancer and the benefits of cervical cancer screenings ${ }^{15,17}$. The results of previous studies indicate that in Ghana, knowledge about cervical cancer and HPV is extremely low even among highly educated women $^{18,19}$. There is a lack of research regarding the psychological barriers, and specific cultural barriers to seeking cervical cancer screening among Ghanaian women with cancer and without cancer.

The purpose of this study was to use a mixed methods approach to investigate psychological barriers among Ghanaian women including determining their knowledge of cervical cancer risk factors, and identifying specific cultural beliefs and cultural health behaviors that may act as impediments to cervical cancer screening. This is a novel study especially since we included women with cancer in our sample. Through the use of semi-structured interviews, we were able to collect quantitative and qualitative data that allowed us to identify gaps in knowledge about cancer signs and symptoms, common misconceptions about cervical cancer, and cultural taboos related to healthcare practices that may impede access to cervical cancer screening. The results of this study can be used to inform the development of culturally relevant cervical cancer education interventions targeted at Ghanaian women with and without cancer.

\section{Methods}

\section{Participant recruitment}

This study was conducted in Kumasi, the second largest city in Ghana. Kumasi is an urban area with a population of more than 1.1 million people. Ghanaian women 18 years old and older, who could speak, hear, and comprehend English or Twi (the most common native language in Kumasi), were recruited to participate in this study.

African Health Sciences Vol 13 Issue 4 December 2013
A purposive sampling plan was used to select participants to guarantee that women with cancer were included in the sample. Women with cancer were recruited from the oncology unit of a large public hospital and from a private hospital that specialized in breast cancer treatment. Women without cancer were recruited from public areas in Kumasi such as the market, pharmacies, trotro (public transportation) stops, and from the campus of the university. All women who completed an interview were informed of the purpose of the study. Verbal consent, not written consent, obtained from each participant before each interview. The interviews were conducted over a oneweek period during the summer of 2009 .

\section{The Instrument}

The theoretical framework used to design this study was based on constructs from two domains of the PEN-3 Model that was developed by Airhihenbuwa ${ }^{20}$. Based on the Educational Diagnosis domain of the PEN-3 model, an individual's perceptions about a health issue can facilitate or hinder a health behavior such as cervical cancer screening. Therefore, we used openended questions to identify the women's knowledge, attitudes, and beliefs about cervical cancer and cervical cancer screening. We also sought to identify enablers (i.e. spouses) that may serve as barriers to cervical cancer screening. Finally, based on the Cultural Appropriateness domain, we sought to identify negative health behaviors that may delay seeking preventive cervical cancer screenings.

A parallel mixed methods design was used to collect qualitative and quantitative data simultaneously. A semi-structured interview guide was used to collect the data. The interview guide consisted of 43 items, including items to assess the participants' knowledge of general cancer risk factors, knowledge of general signs and symptoms of cancer, barriers to mammography, and barriers to cervical cancer screening. Ten opened-ended questions were used to collect qualitative data, including data about the participants' beliefs about cervical cancer risk factors, the impact of a health care provider's gender on cervical cancer screening behavior, and a husband's reaction to his spouses' cancer diagnosis. The variables that will be discussed in this report are cervical cancer screening history, knowledge of general cancer signs and symptoms, psychological barriers to cervical cancer screening, cultural beliefs about cervical cancer risk factors, and the stigmatization of women with cervical cancer. The results of the analysis of the remaining variables will be published in a subsequent manuscript. Ghanaian professors and research assistants from the Faculty of Pharmacy and Pharmaceuticals at the 
Kwame Nkrumah University of Science and Technology reviewed the interview guide to establish content validity and to ensure that the items in the guide were written in a culturally appropriate manner. Modifications to the interview guide were made as needed.

Four Ghanaian research assistants who were proficient in English and Twi were trained to conduct the interviews. The interviews were conducted in the language that the participant preferred. In most cases, the interviews were conducted in Twi. The interviewers translated and transcribed the participants' responses into English.

The Statistical Package for Social Science (SPSS) version 16 was used to analyze the quantitative data. Nvivo 8 was used to store and organize the qualitative data. Indexed coding was used to develop a codebook of common themes that emerged from the qualitative data. Qualitative counting was used to identify the most common themes that occurred across the interviews.

\section{IRB Approval}

This study received approval from the Institutional Review Board at Kwame Nkrumah University of

Table 1: Demographic characteristics of study participants $(\mathbf{N}=220)$

\begin{tabular}{|c|c|c|}
\hline Demographic characteristic & $\begin{array}{l}\text { Current Cancer } \\
\text { patients }(n=49) \\
\text { Frequency }(\%)\end{array}$ & $\begin{array}{l}\text { Non-patients } \\
(\mathrm{n}=171) \\
\text { Frequency }(\%)\end{array}$ \\
\hline \multicolumn{3}{|l|}{ Age range, (years) } \\
\hline $18-24$ & $5(10.2)$ & $75(45.5)$ \\
\hline $25-35$ & $5(10.2)$ & $54(32.7)$ \\
\hline $36-45$ & $18(36.7)$ & $16(9.7)$ \\
\hline $46-55$ & $12(24.5)$ & $11(6.7)$ \\
\hline $56-65$ & $6(12.2)$ & $9(5.5)$ \\
\hline $66-75$ & $3(6.1)$ & $0(0)$ \\
\hline \multicolumn{3}{|l|}{ Education } \\
\hline No formal education & $6(12.2)$ & $9(5.4)$ \\
\hline Primary school & $8(16.3)$ & $10(6.0)$ \\
\hline Junior high school & $12(24.5)$ & $50(29.8)$ \\
\hline Senior high school & $12(24.5)$ & $34(20.2)$ \\
\hline Tertiary ins titution & $10(20.4)$ & $52(31.0)$ \\
\hline Graduate or professional school & $1(2.0)$ & $13(7.7)$ \\
\hline \multicolumn{3}{|l|}{ Martial Status } \\
\hline Single, not married & $11(22.4)$ & $109(64.9)$ \\
\hline Married & $27(55.1)$ & $50(29.8)$ \\
\hline Divorced & $7(14.3)$ & $4(2.4)$ \\
\hline Widowed & $4(8.2)$ & $5(3.0)$ \\
\hline \multicolumn{3}{|l|}{ Health Insurance Status } \\
\hline Insured & $47(97.9)$ & $127(75.6)$ \\
\hline Uninsured & $1(2.1)$ & $41(24.4)$ \\
\hline \multicolumn{3}{|l|}{ Ever had a mammogram } \\
\hline Yes & $37(75.5)$ & $24(14.3)$ \\
\hline No & $12(24.5)$ & $144(85.7)$ \\
\hline $\begin{array}{l}\text { Ever had a cervical cancer } \\
\text { screening }\end{array}$ & & \\
\hline Yes & $8(16.3)$ & $4(2.4)$ \\
\hline No & $41(83.7)$ & $164(97.6)$ \\
\hline
\end{tabular}

Science and Technology. The interviewers read the informed consent letter to the participants and obtained verbal, not written, consent prior to the beginning every interview.

\section{Results}

\section{Participant characteristics}

The study sample (n) consisted of 220 Ghanaian women including 49 women who were being treated for cancer at the time of the study, and 171 noncancer patients. The majority of the participants who had cancer were between the ages of 35 and 74 $(79.6 \%)$ and the majority of the participants without cancer $(78.2 \%)$ were between the ages of 18 and 35 . The majority of the participants with cancer and the participants without cancer had at least completed junior high school $(71.5 \%$ and $88.6 \%$, respectively). Almost all of the participants with cancer $(97.9 \%)$ reported having health insurance. In contrast, $75.6 \%$ of the participants without cancer had health insurance. The demographic characteristics of our sample are displayed in table 1. 
Cervical cancer screening history

Only $8(16 \%)$ of the participants with cancer, and 4 $(2.3 \%)$ of the participants without cancer reported that they had obtained a cervical cancer screening in the past. We asked those respondents who had been screened why they had obtained a cervical cancer screening. As illustrated by the statement below, the majority of the respondents who had been screened indicated that a doctor had recommended it as a diagnostic test, "Because it was prescribed by a doctor" (cancer patient).

Two respondents indicated that they had chosen to get a cervical cancer screening as a means of prevention. This statement below from a noncancer patient explains why she obtained a cervical cancer screening, "Just wanted to have a check" (noncancer patient).
Knowledge of general Cancer risk factors

A Chi-square test of independence indicated that there was no significant association between cancer treatment status and the knowledge of four orfive common signs of cancer: changes in bowel habits; difficulty swallowing and indigestion; unexplained fever; unexplained weight loss; and swellings having emerged any place on the body (table 2). All participants had relatively low knowledge of these cancers signs and symptoms. A Chi-square test of independence indicated that non-cancer patients were more likely to know that abnormal bleedings or excretions from any place on the body was a sign of cancer than women with cancer, $\chi 2(1, \mathrm{n}=217)=5.07, \mathrm{p}=0.024 \mathrm{phi}=$ 0.153 .

Table 2: Respondents knowledge of general signs and symptoms of cancer

\begin{tabular}{|c|c|c|c|}
\hline Which of the following are signs of cancer? & $\begin{array}{l}\text { Current } \\
\text { Cancer } \\
\text { patients } \\
\mathrm{n}(\%)\end{array}$ & $\begin{array}{c}\text { Non-patients } \\
\text { n }(\%)\end{array}$ & p-value \\
\hline ... changes in bowel habits & $4(8.2)$ & $27(16.1)$ & 0.164 \\
\hline ... difficulty swallowing and indigestion & $11(22.4)$ & $33(19.6)$ & 0.667 \\
\hline $\begin{array}{l}\text {.. abnormal bleeding or excretions from any place } \\
\text { on the body }\end{array}$ & $12(24.5)$ & $71(42.3)$ & 0.024 \\
\hline ... unexplained fever & $27(55.1)$ & $71(42.3)$ & 0.112 \\
\hline$\ldots$ unexplained weight loss & $25(51.0)$ & $77(45.8)$ & 0.522 \\
\hline ... swellings have emerged any place on the body & $23(46.9)$ & $168(57.1)$ & 0.207 \\
\hline
\end{tabular}

\section{Cultural beliefs about Cervical cancer risk factors}

The known risk factors for cervical cancer include infection with a high-risk strain of HPV, Chlamydia infection, sexual intercourse with multiple partners, the early initiation of sexual intercourse, multiple full-term pregnancies, smoking tobacco, the long-term use of oral contraceptives, and immunosuppression ${ }^{1,3,4}$. An open-ended question was used to collect data about the participants' beliefs about the causes of cervical cancer. Examples of the most common responses are provided in table 3.
Table 3: The most common beliefs about Cervical Cancer risk factors reported by the study participants

\begin{tabular}{ll}
\hline Cervical cancer risk factor & $\begin{array}{l}\text { Number of } \\
\text { times stated } \\
\text { by participa- } \\
\text { ants }(\boldsymbol{n})\end{array}$ \\
\hline White (Candidiasis) and other & 18 \\
vaginal infections & \\
Chemical or other substances applied 14 \\
to the vagina \\
Multiple sexual partners & 12 \\
Genetics/Family History & 10 \\
Early Initiation of sex & 7 \\
Too much sex & 6 \\
Cigarette smoking & 5 \\
Eating Habits & 5 \\
Poor Hygiene & 4 \\
Contraceptive method & 4 \\
Promiscuity & 4 \\
\hline
\end{tabular}


Many of the participants said that they had "no idea" about the cause of cervical cancer. Only one participant, a non-cancer patient, stated that cervical cancer was caused by "the human papillomavirus." The respondents with higher levels of education were more likely to provide correct, or partially correct responses than women with lower levels of education. This statement from a non-cancer patient is an illustration of a partially correct response "being sexually active at a very young age, genetics, some contraceptive methods."

Many participants had additional misconceptions about the risk factors for cervical cancer. For example, several participants thought that having abortions, inserting herbs or other substances into the vagina or exposing the vagina to chemicals caused cervical cancer. For example a non-cancer patient stated that cervical cancer was caused by, "Inserting local herbs in vagina, cervical problems which are left untreated." A cancer patient stated that cervical cancer was caused by, "Insertion of unwanted materials into the vagina." And a noncancer patient stated that cervical cancer occurred after, "washing the vagina with chemicals unnecessarily." Several participants also thought that poor hygiene was a risk factor for cervical cancer. The following statement from a participant without cancer is an example of this belief, "washing your vagina with dirty hands [causes cervical cancer]."

Many participants also incorrectly believed that cervical cancer was caused by poor eating habits, infertility, immoral lifestyles (i.e. drinking alcohol, using drugs), wounds from cuts, and excessive amounts of sexual intercourse. For example, a participant with cancer stated that cervical was caused by "too much sex making and STD, and applying chemicals to your private part."

A few participants stated that they believed that cervical cancer had a spiritual cause or was associated with witchcraft. This quote, from a noncancer patient, illustrative these beliefs, "It is spiritual."

\section{Screenings performed by a male doctor}

The participants were asked if they would be willing to get a cervical cancer screening if they knew that a male doctor would perform the screening. The gender of the healthcare provider performing the screening appeared to be a barrier for some of the participants. Approximately 26\% $(n=42)$ of the participants who were not being treated for cancer stated that they would not have a screening that if it was performed by a male doctor. The cancer patients were more likely than the non-cancer patients to state that they would not have a problem obtaining a cervical cancer screening from a male doctor. This statement from a cancer patient illustrates this belief: "Yes [I allow a male doctor to perform the screening], the screening is most important because cancer is quite a big issue."

In some Ghanaian communities, a man plays a significant role in the healthcare decisions of his wife ${ }^{21,22}$. Therefore, we asked participants if their husband would allow them to have a cervical cancer screening performed by a male doctor. Participants without cancer were more likely to indicate that their husbands would not allow them to be screened or would not be comfortable with them being screened by a male doctor. These statements from women without cancer illustrate participants' beliefs about why their husbands would not allow them to get screened by a male doctor:

"No, traditionally not right." (Non-cancer patient)

"No, my husband has paid my bride price so no man should see my nakedness." (Noncancer patient)

"Don't think so, men are jealous." (Noncancer patient)

\section{Husband's reaction to cancer diagnosis}

The participants were asked to share their opinions about what a man would do if his wife was diagnosed with cervical cancer. The responses to this question were mixed. The most common responses indicated that a husband's reaction to the cancer diagnosis would depend on the man. For example, a cancer patient stated, "He might support her through the disease or leave her, it depends on the man."

As exemplified by this statement from a cancer patient, "It is the husband's duty to care for the woman," some participants believed that a husband would care for and support his wife if she was diagnosed with cancer. Several cancer patients also stated that a husband would provide financial and emotional support for his wife. For example, a non-cancer patient stated a husband would, "love her, and seek good treatment for her."

Several women stated that a husband might cheat on, abandon, or divorce his wife if is she was diagnosed with cervical cancer. As illustrated below, reasons for these reactions include the husband not 
being able to have sex with his wife, and a husband's belief that his wife developed cancer because she had sex with another man:

"Most men would either leave the woman or cheat. Few will encourage and support the woman." (Non-cancer patient)

"Leave her to marry another woman because sex might not be possible." (Noncancer patient)

"Some will help to see a doctor; some will think that you play double game" (non-cancer patient)

\section{Community members' reaction to women with cervical cancer}

We asked the participants to tell us what people in their communities say about women with cervical cancer. Several participants stated that they did not know what people in their communities say about women with cervical cancer, especially since many women do not disclose their cancer diagnosis. For example, one non-cancer patient stated, "I haven't heard any comment about that before."

Several of the cancer patients and women without cancer said that people in their communities think negatively about women with cervical cancer and people in their communities held stigmatized beliefs about women with cancer. As illustrated by the statements below, the participants indicated that women with cervical cancer were thought of as promiscuous, unsanitary, or cursed by God:

"Classified as evil in society." (non-cancer patient)

"Had been used too much by most men or prostitute." (cancer patient)

"They don't take good care of themselves in terms of personal hygiene." (non-cancer patient)

\section{Discussion}

The age-standardized cervical cancer mortality rate in Ghana is 39.5 per 100,000 women, which is more than twice the global cervical cancer morality rate (15.2 per 100,000 women $)^{1}$. Cervical cancer screening can be obtained in public and private hospitals throughout Ghana ${ }^{10}$. Also, organized cervical cancer screening events have been held in rural areas in Ghana ${ }^{11}$. In developing countries that have resources for cervical cancer screening, the WHO recommends that women should get screened every three years starting at age $25^{24}$. In this study, only 10 participants who were age 25 or older $(4.5 \%)$ reported ever having a cervical cancer screening.

The WHO has suggested that the major barriers to the control of cervical cancer in developing countries, such as Ghana, include women's attitudes, misconceptions, and beliefs about cervical cancer ${ }^{24}$. Therefore, there is a need to determine the non-physical barriers to cervical cancer screening in Ghana. There is a lack of studies that have been conducted to identify the psychological barriers to cervical cancer screening in the target population.

Using the PEN-3 model as the theoretical framework of this parallel mixed methods study, we were able to identify specific intrapersonal and interpersonal psychological barriers to cervical cancer screening for women in Ghana ${ }^{20}$. These barriers included the lack of knowledge of cervical cancer, beliefs in local myths about cervical cancer, stigmatization of women with cervical cancer, misconceptions about the causes of cervical cancer, and the lack of spousal support for cervical cancer screening. The results of this study are consistent with studies that were consistent to the findings of similar studies that were conducted in other developing countries $^{4,25-28}$.

The results of this study add to a small body of published literature about cervical cancer prevention in Ghana ${ }^{18,19,13,20}$. The psychological barriers that were identified in this study must be addressed through culturally relevant health communication interventions. Studies from other developing countries and ethnically diverse groups in the United States have shown that culturally relevant health communication interventions can increase awareness about cancer and cancer prevention, and subsequently lead to an increase in participation in cancer screening ${ }^{20,29,30-32}$. An effective cervical cancer education intervention has the potential to decrease the incidence and mortality rates of cervical cancer in Ghana by increasing knowledge and awareness of cervical cancer screenings, and reducing barriers to cervical cancer screening.

\section{Limitations of the study}

The purposive sampling plan that was used to recruit participants is one limitation of this study. We intended to recruit women with cancer to participate in the study; therefore we could not rely on random sampling methods to select the study participants. In addition, this study was conducted in a large urban 
city and many of the participants were highly educated. Therefore, results of this study may not be generalizable to Ghanaian women in rural areas, and women with low levels of education.

An additional limitation of this study was our lack of data on the specific type of cancer that our participants with cancer were being treated for. Due to the sensitive nature of the topic of this study, we chose not to ask cancer patients to identify the type of cancer that they were being treated for.

\section{Conclusion}

The results of this study indicate that some Ghanaian women hold inaccurate cultural beliefs about cervical cancer risk factors. Consequently, many Ghanaian women may be minimizing their risk for developing the disease. There is a need for culturally appropriate cervical cancer education interventions aimed at addressing these misconceptions and increasing Ghanaian women's knowledge of cancer signs and symptoms, and their perceived risk of cervical cancer and perceived need for cervical cancer screenings.

\section{References}

1. World Health Organization, Information Centre on HPV and Cerival Cancer (HPV) Information Centre. Summary report on HPV and cervical cancer statistics in Ghana 2010. http:// www.who.int/hpvcentre. .

2. Wiredu E, and Armah, H. Cancer mortality patterns in Ghana: A 10-year review of autopsies and hospital mortality. BMC Public Health. June 2006 2006;6(159).

3. Smith JS, Melendy A, Rana RK, Pimenta JM. Age-specific prevalence of infection with human papillomavirus in females: a global review. $J$ Adolesc Health. Oct 2008;43(4 Suppl):S5-25, S25 e21-41.

4. Agurto I, Sandoval J, De La Rosa M, Guardado ME. Improving cervical cancer prevention in a developing country. Int J Qual Health Care. Apr 2006;18(2):81-86.

5. Bosch FX. New opportunities for cancer prevention. Vaccine. 2009;27 Suppl 1:A2-A3.

6. Gaffikin L, McGrath JA, Arbyn M, Blumenthal PD. Visual inspection with acetic acid as a cervical cancer test: accuracy validated using latent class analysis. BMC Med Res Methodol. 2007;7:36.

7. Scarinci IC, Garcia FA, Kobetz E, et al. Cervical cancer prevention: new tools and old barriers. Cancer. Jun 1 2010;116(11):2531-2542.
8. Wright TC, Jr., Blumenthal P, Bradley J, et al. Cervical cancer prevention for all the world's women: new approaches offer opportunities and promise. Diagn Cytopathol. Dec 2007;35(12):845-848.

9. Luciani S, Jauregui B, Kieny C, Andrus JK. Human Papillomavirus vaccines: new tools for accelerating cervical cancer prevention in developing countries. Immunotherapy. 2009;1(5):795-807.

10. Blumenthal P, Gaffikin, L., Deganus, S., Lewis, R., Emerson, M., and Adadevoh, S. Cervical cancer prevention: Safety, acceptability, and feasibility of a single-visit approach in Accra, Ghana. Am J Obstet Gynecol. April 2007 2008;196:407.e401-407.e409.

11. Sanghvi H, Limpaphayom KK, Plotkin M, et al. Cervical cancer screening using visual inspection with acetic acid: operational experiences from Ghana and Thailand. Reprod Health Matters. Nov 2008;16(32):67-77.

12. Ohene-Yeboah MaA, E., Amaning E. Spectrum of Complaints Presented at a Specialist Breast Clinic in Kumasi, Ghana. Social Science \& Medicine. September 2008;42(3):110-112.

13. Reichenbach L. The politics of priority setting for reproductive health: breast and cervical cancer in Ghana. Reprod Health Matters. Nov 2002;10(20):47-58.

14. Tabi M, Powell., M., and Hodnicki, D. Use of tradtional healers and modern medicine in Ghana. International Nursing Review. 2006;53:5258.

15. Lingwood R, Boyle, P., Milburn, A., Ngoma, T., Arbuthnott, J., McCaffrey, R., Kerr, S., and Kerr, D. The challenge of cancer control in Africa. Nature Reviews Cancer. May 2008 2008;8:398-403.

16. Affrriye K. Ghana Health Sector's 2004 Annual Program of Work Accra: Ghana Ministry of Health;2004.

17. Mayhew S, and Adjei, S. Sexual and reproductive health: challenges for priority setting in Ghana's health reforms. Health Policy and Planning. 2004;19(Suppl. 1):i50-i61.

18. Abotchie PN, Shokar NK. Cervical cancer screening among college students in ghana: knowledge and health beliefs. Int J Gynecol Cancer. Apr 2009;19(3):412-416.

19. Adanu R. Cervical Cancer Knowledge and Screening in Accra, Ghana. Journal of Women's Health \& Gender-Based Medicine. 2002;11(6):487488.

African Health Sciences Vol 13 Issue 4 December 2013 
20. Airhihenbuwa C, Okoro T. Toward EvidenceBased and Culturally Appropriate Models for Reducing Global Health Disparities: An Africanist Perspective. In: Wallace BC, ed. Toward equity in health: A new global approach to health disparities. New York: Springer Publications; 2008.

21. Blanchard CM, Denniston MM, Baker F, et al. Do adult change their lifestyle behaviors after cancer diagnosis? American Journal of Health Behavior. 2003;27(3):246-256.

22. Bierlich B. The problem of money: African agency and western medicine in northern Ghana. New York: Berghahn Books; 2007.

23. Boateng J, Flanagan C. Women's access to health care in Ghana: effects of education, residence.

24. World Health Organization. Comprehensive Cervical Cancer Control: A guide to essential practice. Geneva: World Health Organization; 2006.

25. Murthy P, Smith CL. Women's global health and buman rights. Sudbury, Mass.: Jones and Bartlett Publishers; 2010.

26. Othman NH, Rebolj M. Challenges to cervical screening in a developing country: The case of Malaysia. Asian Pac. J. Cancer Prev. 2009;10(5):747752.

27. Lin S-J. Factors influencing the uptake of screening services for breast and cervical cancer in Taiwan. $J$ Soc Promot Health. 2008;128(6):327-334.

28. Agurto I, Bishop A, Sánchez G, Betancourt Z, Robles S. Perceived barriers and benefits to cervical cancer screening in Latin America. Prev Med. 2004;39(1):91-98.

29. Holt CL, Scarinci IC, Debnam K, et al. Spiritually based intervention to increase colorectal cancer awareness among african americans: intermediate outcomes from a randomized trial. J Health Commun. 2012;17:1028-49.

30. Scarinci IC, Bandura L, Hidalgo B, Cherrington A. Development of a theory-based (PEN-3 and Health Belief Model), culturally relevant intervention on cervical cancer prevention among Latina immigrants using intervention mapping. Health Promot Pract. 2012;13:29-40. doi:10.1177/1524839910366416.

31. Hawkins RP, Kreuter M, Resnicow K, Fishbein M, Dijkstra A. Understanding tailoring in communicating about health. Health Education Research. 2008;23:454-466.

32. Baskin ML, Resnicow K, Campbell MK. Conducting health interventions in black churches: a model for building effective partnerships. Ethn Dis. 2001;11:823-33. 